$\bigvee$ Workshop de Pesquisa em Manufatura

ANAIS DO V WORKSHOP DE PESQUISA EM MANUFATURA 
$\checkmark$ Workshop de Pesquisa em Manufatura

ANAIS DO V WORKSHOP

DE PESQUISA EM MANUFATURA 
Editora Omnis Scientia

ANAIS DO V WORKSHOP DE PESQUISA EM MANUFATURA

Volume 1

$1^{a}$ Edição

TRIUNFO - PE

2021 
PROMOÇÃO:

Programa de Pós-Graduação em Engenharia Mecânica (PPGEMec/UFSCar)

Apresentadores (as):

Adailton Gomes Pereira

Alana Carla Miranda Araújo

Bruna Oliveira da Silva

Bruno Alexandre Roque

Caique de Castro Gonçalves

Cassiano da Silva Tavares

Cristie Luis Kugelmeier

Gustavo Roberto dos Santos

Henrique A. C. Durello

Hugo Emanoel de Andrade Costa

Jonatan Augusto da Silva

Leones Contini Junior

Marco Gabriel Lorenzoni

Matheus Luis Manfredo

Paulo De Tarso Durigan

Rafael Fernando Teixeira

Talia Gibim

Tony Emerson Marim.

Participantes do painel:

Carlos Eiji Hirata Ventura

Danielle Cristina Camilo Magalhães

Rodrigo da Silva ("PPGEMec: Presente e futuro").

\section{Palestrante:}

Yayue Pan - University of Illinois Chicago ("Field-assisted Photopolymerization-based Additive Manufacturing for Productions of Multi-Functional Materials and Devices")

\section{Comitê científico:}

Alexandre Tácito Malavolta

Carlos Eiji Hirata Ventura

Danielle Cristina Camilo Magalhães

Flávio Yukio Watanabe

Marcos Roberto Monteiro

Rodrigo da Silva

Sérgio Henrique Evangelista.

\section{Comissão organizadora:}

Adailton Gomes Pereira 
Armando Ítalo Sette Antonialli

Bruna Oliveira da Silva

Edson Bruno Lara Rosa

Sidney Bruce Shiki.

\section{Imagem de Capa}

Freepik

\section{Edição de Arte}

Vileide Vitória Larangeira Amorim

\section{Revisão}

Os autores

\section{(C) $(1) \Theta \Theta$}

Este trabalho está licenciado com uma Licença Creative Commons - Atribuição-NãoComercialSemDerivações 4.0 Internacional.

O conteúdo abordado nos artigos, seus dados em sua forma, correção e confiabilidade são de responsabilidade exclusiva dos autores. 
Dados Internacionais de Catalogação na Publicação (CIP) (eDOC BRASIL, Belo Horizonte/MG)

Workshop de Pesquisa em Manufatura (5 : 2021)

W912a Anais do [...] / V Workshop de Pesquisa em Manufatura, 10

dezembro 2021. - Triunfo, PE: Omnis Scientia, 2021.

$52 \mathrm{p}$.

Formato: PDF

Requisitos de sistema: Adobe Acrobat Reader

Modo de acesso: World Wide Web

ISBN 978-65-88958-78-0

DOI 10.47094/978-65-88958-78-0

1. Engenharia mecânica - Brasil - Congressos. 2. Manufaturas. I. Título.

CDD 621.7

Elaborado por Maurício Amormino Júnior - CRB6/2422

\section{Editora Omnis Scientia}

Triunfo - Pernambuco - Brasil

Telefone: +55 (87) 99656-3565

$\underline{\text { editoraomnisscientia.com.br }}$

contato@editoraomnisscientia.com.br

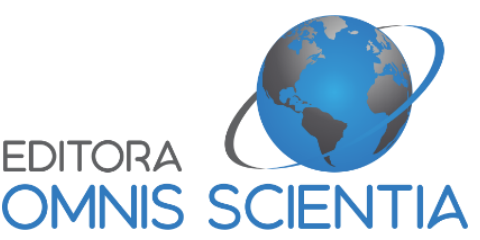




\section{PREFÁCIO}

A exemplo do ensino e da extensão, a pesquisa no âmbito da Engenharia Mecânica apresenta-se bastante ampla e diversificada. A ramificação mais canônica dessa modalidade de engenharia aponta para três áreas fundamentais: Projeto de Sistemas Mecânicos, Ciências Fluidotérmicas e Processos de Fabricação. Outras subdivisões, como Dinâmica e Vibrações, Mecatrônica e Metrologia, flutuam entre as três grandes áreas. Sendo assim, a nucleação e o crescimento do Grupo de Pesquisa em Manufatura Inteligente (GPMI), devidamente registrado no Diretório dos Grupos de Pesquisa do CNPq e reconhecido pela UFSCar, têm sido salutar no sentido da consolidação do Departamento de Engenharia Mecânica (DEMec) e do curso de graduação em Engenharia Mecânica. Da mesma forma, O GPMI se mostra essencial enquanto alicerce para o recém-criado curso de mestrado acadêmico dentro do Programa de PósGraduação em Engenharia Mecânica (PPGEMec), conferindo-lhe vocação e identidade. Finalmente, a comunicação e a difusão dos resultados obtidos pelo grupo propiciam o alinhamento do mesmo ao lema da UFSCar: excelência acadêmica e compromisso social. O objetivo geral do "V Workshop de Pesquisa em Manufatura" consiste em disseminar os trabalhos em andamento dentro do Grupo de Pesquisa em Manufatura Inteligente (GPMI) e de outros grupos afins à área de Manufatura, promovendo discussões

profícuas e de alto nível com o público interno e externo à universidade. Como objetivos específicos, destacam-se a exposição dos estudantes de graduação e pós-graduação a um ambiente de conferência científica sem sair de casa e a oportunidade de contar com convidados com grande potencial de contribuição a essa área do conhecimento. O evento foi realizado ao longo do dia 10 de dezembro de 2021, de forma online, de maneira a viabilizar e fomentar a participação de uma parcela significativa de estudantes. 


\section{SUMÁRIO}

INFLUÊNCIA DA SENSIBILIDADE DE PARÂMETROS DOS MODELOS DE AVRAMI NAS CINÉTICAS DE RECRISTALIZAÇÃO SOBRE O TAMANHO DE GRÃO EM UM PROCESSO DE LAMINAÇÃO A QUENTE DE LIGA DE AÇO SAE 4140..

SIMULAÇÃO NUMÉRICA DA EXTRUSÃO EM CANAL ANGULAR COM TORÇÃO (ECA-T): INFLUÊNCIA DA GEOMETRIA NA DEFORMAÇÃO.

OTIMIZAÇÃO TOPOLÓGICA APLICADA NO CONTEXTO DE PRÓTESES HUMANAS. .15

PROCESSO DE DOBRAMENTO A FRIO DE CHAPAS FINAS METÁLICAS: ANÁLISES POR ELEMENTOS FINITOS, EXPERIMENTOS E MODELO MATEMÁTICO PARA COMPENSAÇÃO DO RETORNO ELÁSTICO.

FORÇAS NA ESTAMPAGEM INCREMENTAL DE CHAPA FINA METÁLICA POR MEIO DE EXPERIMENTOS E ANÁLISES POR ELEMENTOS FINITOS.

MODELAGEM DE CURVAS DE FLUXO PLÁSTICO DE UM AÇO BIFÁSICO UTILIZANDO INTELIGÊNCIA ARTIFICIAL

APLICAÇÃO DA TECNOLOGIA DE MACHINE LEARNING PARA AVALIAÇÃO DA QUALIDADE DE PEÇAS IMPRESSAS EM 3D...

ESTUDO DA PREVISIBILIDADE DO ERRO DE TRANSMISSÃO DE ENGRENAGENS DE DENTES RETOS PARA PROJETO DE MODIFICAÇÕES DE MICROGEOMETRIA...........................26

CONTRIBUIÇÃO DO PRÉ-AQUECIMENTO DO SUBSTRATO NA PREVENÇÃO DE TRINCAS EM REVESTIMENTOS GERADOS POR LASER CLADDING.

AVALIAÇÃO DA INFLUÊNCIA DA VELOCIDADE DE CORTE SOBRE O ESTADO DE SUPERFÍCIE NO TORNEAMENTO DA LIGA TI-6AL-4V ELI.

INFLUÊNCIA DO TAMANHO DO GRÃO DO REBOLO NA QUALIDADE SUPERFICIAL DO INSERTO DE CORTE DE METAL DURO RETIFICADO.

INVESTIGAÇÃO DOS MECANISMOS DE REMOÇÃO DE MATERIAL NA RETIFICAÇÃO DE INSERTOS DE CORTE.

SHUNT PIEZOELÉTRICO PARA CONTROLE PASSIVO DE CHATTER NO PROCESSO DE TORNEAMENTO DE LIGAS DE TITÂNIO.

AVALIAÇÃO DE TEXTURAS INDUZIDAS POR VIBRAÇÃO NO TORNEAMENTO DURO DE UM AÇO FERRAMENTA.

INVESTIGAÇÃO DA INFLUÊNCIA DO CONTROLE TÉRMICO ATIVO SOBRE A RESISTÊNCIA MECÂNICA E QUALIDADE SUPERFICIAL DE PEÇAS IMPRESSAS PELA TÉCNICA FPM. 
ATUALIZAÇÃO DE MÁQUINAS-FERRAMENTA ANTIGAS ATRAVÉS DE INSTRUMENTAÇÃO DE BAIXO CUSTO: UM ESTUDO SOBRE O EFEITO DO MONITORAMENTO DO PROCESSO DE TORNEAMENTO NA QUALIDADE SUPERFICIAL .46 AVALIAÇÃO MICROESTRUTURAL DO AÇO INOXIDÁVEL LEAN DUPLEX 2404 APÓS SIMULAÇÕES DE CICLOS TÉRMICOS DE ZONAS TERMICAMENTE AFETADAS REALIZADAS POR GLEEBLE. . .48 AVALIAÇÃO DA INFLUÊNCIA DO ACABAMENTO SUPERFICIAL NA RESISTÊNCIA À CORROSÃO DO AÇO 1020 EM BIODIESEL E SUAS MISTURAS COM ÓLEO

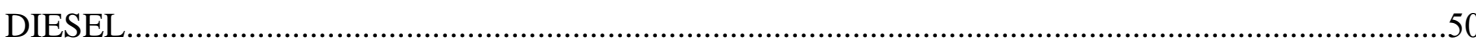




\title{
INVESTIGAÇÃO DA INFLUÊNCIA DO CONTROLE TÉRMICO ATIVO SOBRE A RESISTÊNCIA MECÂNICA E QUALIDADE SUPERFICIAL DE PEÇAS IMPRESSAS PELA TÉCNICA FPM
}

\author{
Caique de Castro Gonçalves \\ Alexandre Tácito Malavolta \\ Universidade Federal de São Carlos, São Carlos, Brasil \\ caiquecastro@estudante.ufscar.br \\ malavolta@ufscar.br
}

\begin{abstract}
Resumo: Atualmente, o processo de manufatura aditiva se tornou uma prática comum em diversos setores industriais em função de sua capacidade de impressão em larga escala, alta produtividade de peças com geometria complexas. Dentre as diversas técnicas de manufatura aditiva, destaca-se a técnica FPM (Fused Pellet Modeling) na qual materiais poliméricos na forma de grãos (pellets) são utilizados. Um dos grandes desafios da impressão de componentes funcionais pelo processo FPM refere-se à qualidade superficial e a resistência mecânica das peças produzidas. Tal problemática tende a ser mais acentuada no caso da impressão de grandes volumes, uma vez que os gradientes térmicosassociados tendem a ser maiores. Neste contexto, o presente projeto de pesquisa propõe a investigação da influência docontrole térmico ativo da mesa de impressão sobre a resistência mecânica e qualidade superficial de peças impressas pela técnica FPM de materiais poliméricos em ABS e PLA. Para tal, será realizado o desenvolvimento de uma mesa deimpressão com controle ativo de temperatura para compor uma célula de manufatura de impressão $3 D$ para fabricaçãode produtos de grande porte (com volume de até $1 \mathrm{~m}^{3}$ ). Nesta célula, o material polimérico em grãos (pellets) será processado em uma extrusora monorosca a ser manipulada por um braço robótico industrial.
\end{abstract}

Palavras-chaves: manufatura aditiva, controle de temperatura, resistência mecânica.

\section{INTRODUÇÃO}

Nas últimas décadas, a manufatura aditiva tornou-se um processo de fabricação de grande interesse para indústria dadas suas vantagens na produção de geometrias complexas e utilização de diferentes materiais de fabricação. Dentre as diversas técnicas de manufatura aditiva, destaca-se a técnica FPM (Fused Pellet Modeling) na qual materiais poliméricos na forma de grãos (pellets) são utilizados. Uma característica fundamental do processo FPM é o seu potencial para fabricar peças com propriedades controladas localmente (porosidade, densidade e propriedades mecânicas) de acordo com Sun et al. (2008) apud Li et al., (2002). Um dos grandes desafios da impressão de componentes funcionais pelo processo FPM refere-se à qualidade superficial e a resistência mecânica das peças produzidas. Tal problemática tende a ser mais acentuada no caso da impressão de grandes volumes, uma vez que os gradientes térmicos associados tendem a ser maiores. $\mathrm{O}$ histórico de temperatura das interfaces desempenha um papel importante para determinar a qualidade da ligação e, portanto, a propriedades mecânicas do produto final, conforme Sun et al. (2008) e Brett et. al (2017). Neste contexto, o presente projeto de pesquisa propõe uma investigação da influência do controle térmico ativo da mesa de impressão sobre a resistência mecânica e qualidade superficial de peças impressas pela técnica FPM de materiais poliméricos em ABS e PLA. Para tal, será realizado o desenvolvimento de uma mesa de impressão com controle ativo de temperatura para compor uma célula de manufatura de impressão 3D composta por um braço robótico no qual será acoplado uma extrusora monorosca para fabricação de produtos de grande porte, conforme ilustrado na Figura 1.

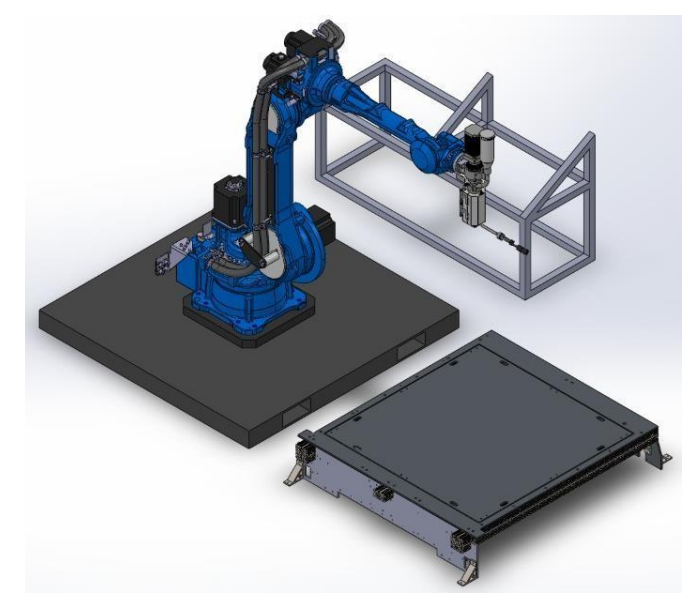

Figura 1: Célula de manufatura para impressão 3D.

\section{MATERIAIS E MÉTODOS}

Inicialmente serão elaborados projetos conceituais de mesas de impressão com controle ativo de temperatura. Será considerado o uso de mantas térmicas para o aquecimento da base da mesa trabalhando em conjunto com um controle do tipo PID para manutenção das temperaturas de interesse como mostrado na Figura 2. 


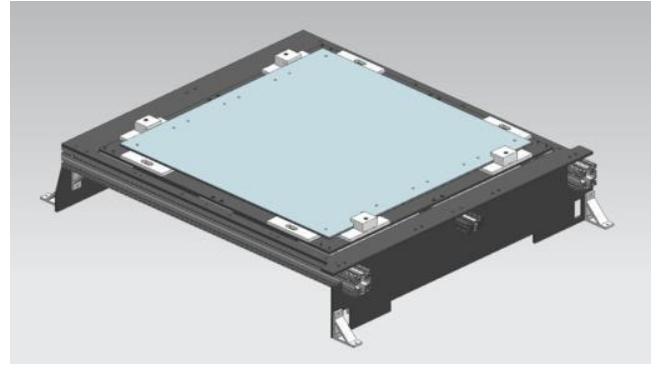

Figura 2: Conceito inicial da mesa de impressão 3D.

Estas configurações preliminares serão analisadas, via método dos elementos finitos, para prever os gradientes térmicos da base da mesa e também prever possíveis distorções oriundas de efeitos termo-elásticos. Os resultados obtidos pelas análises deverão orientar a definição da configuração final da mesa para posterior elaboração do projeto final detalhado, fabricação e montagem. Após a montagem da mesa, será realiza a coleta de dados experimentais com emprego de técnicas de planejamento de experimentos (DOE) para impressão de peças com diferentes parâmetros de controle térmico (variando a temperatura de controle da mesa) e em materiais poliméricos diferentes (ABS e PLA). O material polimérico em grãos (pellets) será processado em uma extrusora monorosca a ser manipulada por um braço robótico industrial e depositado sobre a mesa de impressão onde também uma câmera térmica será posicionada próximo a mesa para acompanhar os gradientes de temperatura na peça impressa. Finalmente, as peças produzidas deverão sofrer uma inspeção dimensional, avaliação de sua qualidade de acabamento e testes de resistência mecânica.

\section{RESULTADOS PRELIMINARES}

Espera-se, ao final do projeto de pesquisa que as simulações de elementos finitos de gradientes térmicos da mesa e a análise de DOE corrobore em fornecer resultados de suporte que possam ser utilizados para otimizar a fabricação de peças de grande volume impressas pela técnica FPM minimizando efeitos indesejáveis de baixa resistência mecânica e qualidade superficial.

\section{CONCLUSÃO}

A pesquisa visa comprovar experimentalmente, em conjunto com simulações de elementos finitos, a importância do controle térmico sobre a qualidade do material impresso. Desta forma espera-se que os resultados forneçam parâmetros de impressão que garantam uma melhor qualidade superficial e resistência mecânica de componentes impressos por FPM.

\section{REFERÊNCIAS}

Akande, S.O., 2015. "Dimensional accuracy and surface finish optimization of fused deposition modelling parts using desirability function analysis". Int. J. Eng. Res. Technol, Vol. 4, No. 4, pp. 196-202.

Brett G. Compton, Brian K. Post, Chad E. Duty, Lonnie Love, Vlastimil Kunc, 2017, "Thermal analysis of additive manufacturing of large-scale thermoplastic polymer composites“, Additive Manufacturing (2017), Vol. 17, pp. 77-86.

Sun, Q., Rizvi, G., Bellehumeur, C. e Gu, P., 2008. "Effect of processing conditions on the bonding quality of FDM polymer filaments". Rapid prototyping journal. 
editoraomnisscientia@gmail.com M https://editoraomnisscientia.com.br/ @editora_omnis_scientia https://www.facebook.com/omnis.scientia.9

+55 (87) 9656-3565 


\section{EDITORA

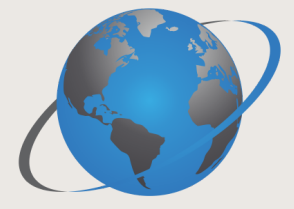 \\ OMNIS SCIENTIA}

editoraomnisscientia@gmail.com M https://editoraomnisscientia.com.br/ $\circledast$

@editora_omnis_scientia (0)

https://www.facebook.com/omnis.scientia.9 f

$$
\text { +55 (87) 9656-3565 }
$$

